\title{
Sustaining Meaningful Patient Engagement Across the Lifecycle of Medicines: A Roadmap for Action
}

\author{
Maria Cavaller-Bellaubi, PharmD ${ }^{1}$ Stuart D. Faulkner, $\mathrm{PhD}^{2}$ (D) B Bryan Teixeira, $\mathrm{PhD}^{3} \cdot$ Mathieu Boudes, $\mathrm{PhD}^{4}$. \\ Eva Molero ${ }^{5} \cdot$ Nicholas Brooke $^{6} \cdot$ Laura McKeaveney $^{7} \cdot$ Jeffrey Southerton ${ }^{8} \cdot$ Maria José Vicente $^{9} \cdot$ Neil Bertelsen $^{10}$. \\ Juan García-Burgos, MD ${ }^{11}$ • Vinciane Pirard, MD ${ }^{12} \cdot$ Kirsty Reid, MD ${ }^{13}$. Elisa Ferrer, PharmD, PhD ${ }^{1}$
}

Received: 9 December 2020 / Accepted: 17 March 2021 / Published online: 10 May 2021

(c) The Author(s) 2021

\begin{abstract}
Background There is increased recognition that incorporating patients' perspectives and insights into the medicines development process results in better health outcomes and benefits for all involved stakeholders. Despite the increased interest and the existence of frameworks and practical recommendations, patient engagement (PE) is not yet considered standard practice. The objective of this work was to provide a roadmap to support systematic change in all stakeholder organisations involved in medicines development across Europe, patients and patient organisations, medicines developers, academia, regulatory authorities, Health Technology Assessment bodies, payers, policy-makers and public research funders, to sustain PE practices. Methods A mixed-methods approach was used by the EU-funded Innovative Medicines Initiative PARADIGM Consortium to co-develop the sustainability roadmap including background work to identify success factors and scenarios for sustainable PE. The roadmap development was based on the Theory of Change concept and populated with findings from (1) interviews with national/ and international institutions with the potential to increase PE uptake by other stakeholders; (2) multi-stakeholder workshops and webinars; and (3) consultations with specific stakeholder groups, Consortium members and a consultative body formed by international PE initiatives.

Results This roadmap sets strategic goals for the PE community to achieve meaningful and systematic PE through changes in the culture, processes and resources of stakeholder organisations. It brings in key PARADIGM outputs to work in a coordinated fashion with existing frameworks and mechanisms to achieve system-wide sustained PE.

Conclusions The roadmap provides a framework for all stakeholders to take collective action within their organisations and across Europe to implement PE in a sustainable manner.
\end{abstract}

Keywords Patient engagement $\cdot$ Medicines development $\cdot$ Roadmap $\cdot$ Sustainability $\cdot$ Call to action

Elisa Ferrer

ferrer.elisa@gmail.com

1 EURORDIS-Rare Diseases Europe, Paris, France

2 Radcliffe Primary Care Building, Radcliffe Observatory Quarter, Woodstock Rd, Oxford OX2 6GG, UK

3 European AIDS Treatment Group (EATG), Düsseldorf, Germany

4 European Patients' Forum (EPF), Chaussée d'Etterbeek, Brussels, Belgium

5 Teamit Research S.L., Barcelona, Spain

6 The Synergist, Brussels, Belgium
7 Novartis International AG, Basel, Switzerland

8 Pfizer Inc, San Diego, CA, USA

9 Aragón Health Sciences Institute, Instituto Aragonés de Ciencias de la Salud (IACS), Zaragoza, Spain

10 Health Technology Assessment International (HTAi)-Patient and Citizen Involvement Interest Group, Berlin, Germany

11 European Medicines Agency (EMA), Amsterdam, The Netherlands

12 Sanofi-Genzyme, Brussels, Belgium

13 European Federation of Pharmaceutical Industries and Associations (EFPIA), Brussels, Belgium 


\section{Introduction}

Incorporating patients' perspectives and insights into the medicines development process is increasingly being recognised and accepted by all stakeholders as an important part of the process of developing innovative medicines that better address patients' unmet needs and priorities [1-3]. Moreover, there is a drive to generate contextualised metrics $[4,5]$ that better capture and demonstrate the value that all stakeholders can derive from patient engagement (PE).

Patients' and their perspectives are increasingly incorporated and valued at different stages of the medicines development process [6] including: research prioritisation (e.g. helping to define unmet needs) [7,8]; clinical programme and clinical trial design [9-11]; early dialogue with regulators [12], health technology assessment (HTA) bodies [13] and competent authorities on pricing and reimbursement ('payers') [14]; as well as during the regulatory approval $[15,16]$ and post-approval phases including HTA evaluation $[17,18]$ and payer decision-making [19]. For this article, medicines development includes the above-mentioned stages. Although acceptance, implementation and regulatory expectations [20] of PE is expanding, it is not yet a standard practice and is still driven by the innovators and early adopters [21] within stakeholder groups, with little widespread urgency towards its full implementation [22].

Whilst multiple frameworks, principles and practical recommendations to operationalise and facilitate the engagement of patients exist, they are often intended for different purposes. Some are designed to facilitate PE in medicines development [9, 23-29] whilst others cover broader engagement in research or the healthcare system $[6,30]$.

This multitude of tools and frameworks allows each stakeholder organisation to use those that best fit their needs; however, they may be short-lived, lack transferability and hinder a consistent approach to sustaining PE practices beyond a few projects. In addition, PE is often approached superficially focusing on the individual's choice and satisfaction rather than providing input into project design [31-33]. Failing to demonstrate that PE provides tangible benefits to all stakeholders may threaten the consolidation of PE practices [4].

The Innovative Medicines Initiative (IMI)-funded PARADIGM (Patients Active in Research And Dialogues for an Improved Generation of Medicines) Consortium ('the Consortium') defined PE as the effective and active collaboration of patients, patient advocates, patient representatives and/ or carers in the processes and decisions within the medicines development lifecycle, along with all other relevant stakeholders when appropriate [34]. The Consortium had two main objectives: (1) to co-develop a comprehensive set of tools and practices to support the integration of patient perspectives into medicines development lifecycle and to demonstrate 'the return on engagement'; and (2) to deliver a strategic sustainability roadmap to support systematic change in stakeholder organisations to make PE common practice. Roadmap implementation relies on the adoption and long-term use of the co-designed resources, in complementarity with existing PE frameworks, and leveraging multi-stakeholder networks. This article describes the development and contents of this strategic roadmap and calls to action to all organisations/institutions involved in medicines development, i.e. patients and patient organisations (POs), medicines developers, academia, regulatory authorities, HTA bodies, payers, policy-makers and public research funders, to achieve meaningful and sustainable PE for better health outcomes.

\section{Roadmap Development Process}

The PE sustainability roadmap was developed by a dedicated multi-stakeholder working group within the Consortium formed by representatives of POs, medicines developers, HTA bodies and academia through a sequence of steps described in Table 1. Detailed methodology has been reported in the corresponding project deliverable [35].

The question 'what makes PE sustainable?'-i.e. what changes are needed for PE to become common practice?drove the initial discussions. Boutin et al. consider that, to ensure routine implementation of PE and its full integration in medicines development, it is critical to establish a culture and processes to overcome existing barriers [36]. Building on the framework proposed by Boutin et al., PARADIGM partners agreed that sustainable PE practices will also require allocation of dedicated financial and human resources, including expertise and capabilities, within stakeholder organisations. The changes needed to achieve sustainable PE were catalogued using three dimensions:

- Culture to drive the necessary behaviour changes to make PE common practice ('the norm').

- Processes to drive stakeholder alignment.

- Resources to mobilise human and financial resources towards sustained PE.

This framework was used to conduct a broad benchmarking exercise across initiatives in the open innovation field (Table 1, step 2) that allowed for the identification of common success factors for long-term survival and operational sustainability. These factors were then categorized according to the three sustainability dimensions (Fig. 1) [37].

Furthermore, the working group aimed to develop scenarios with the greatest potential to accelerate the implementation of PE and make it sustainable (see Table 1, steps 1-4). 


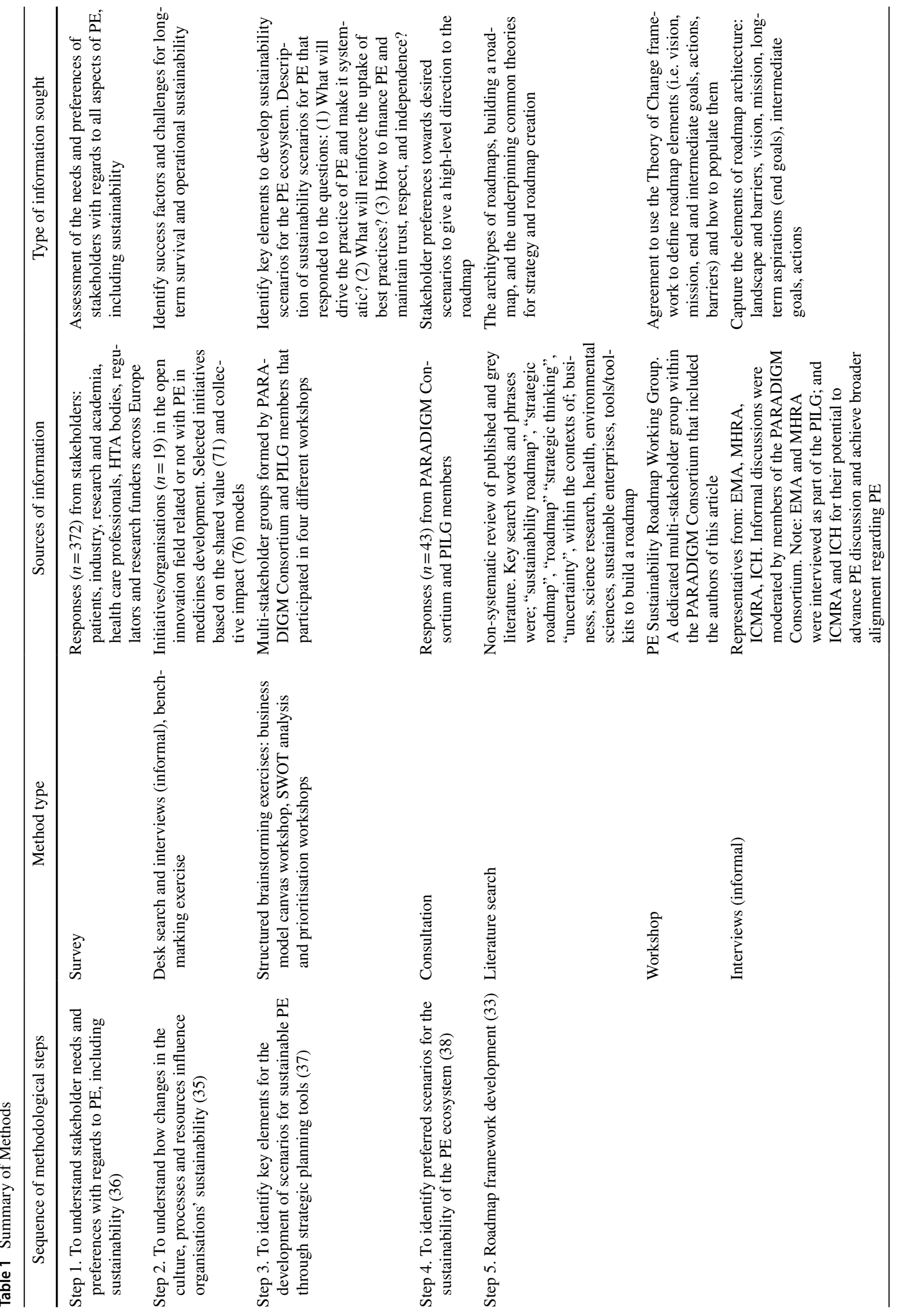




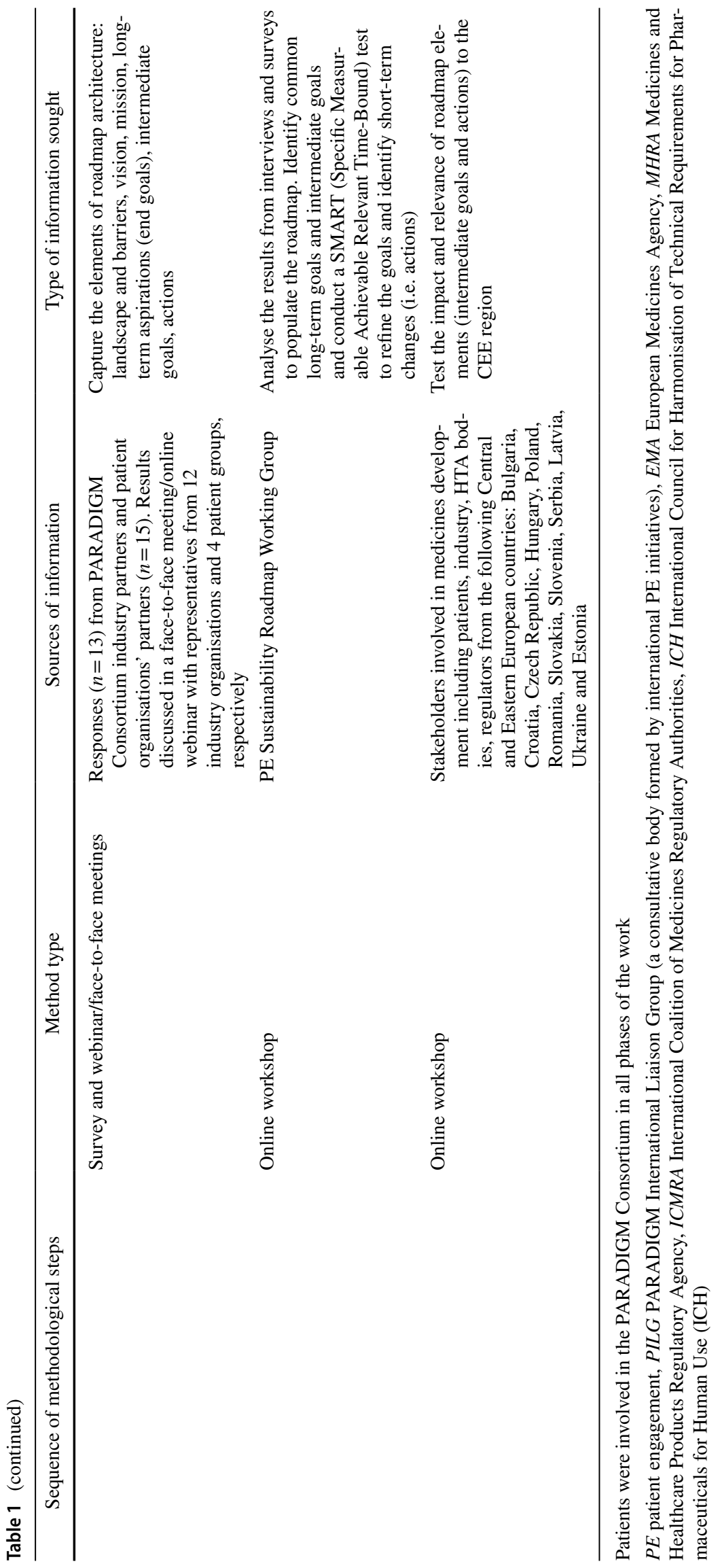




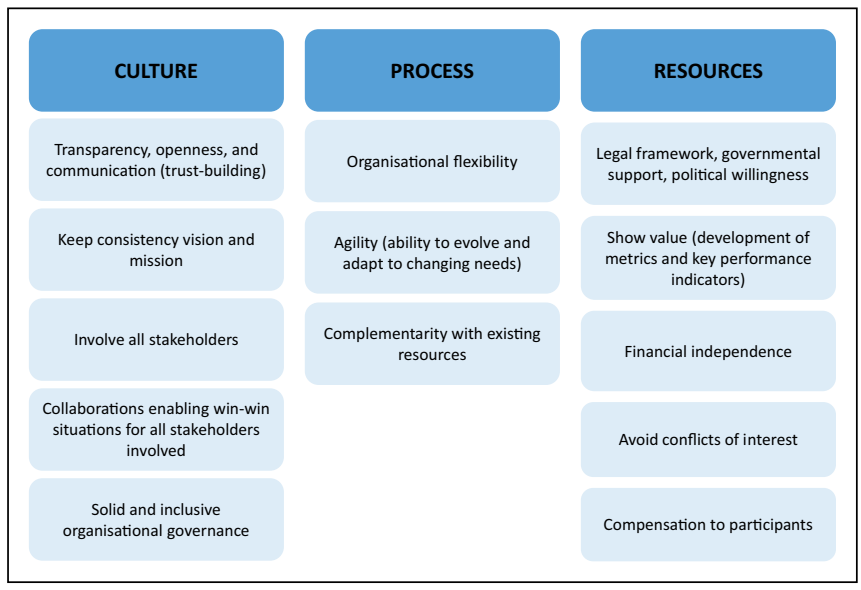

Fig. 1 Common sustainability factors categorized according to the three sustainability dimensions. Source: Informal interviews with initiatives/organisations in the open innovation field related or not with $\mathrm{PE}$ in medicines development

For this task, the group took into account (1) the learnings from a needs and preferences assessment (step 1) [38]; (2) the outcomes of the benchmarking exercise (step 2); (3) the outputs from the different rounds of reflection (step 3); and (4) a consultation process (step 4) [39]. Four major scenarios were suggested (Fig. 2).

For each sustainability scenario an image of how their components could concretely translate in a future PE landscape was described. Whilst PE in medicines development may continue to grow without intervention, one integrated scenario showed the potential to speed up cultural changes and adoption of new processes to sustain PE across the European region. This chosen scenario combines a strong signal from authoritative institutions to make $\mathrm{PE}$ a requirement in medicines development, with a diverse offering of PE services developed by for-profit and non-profit organisations [40]. PE through POs improves engagement opportunities and supports funding to further build patients' engagement capacity, which may be at risk if POs are excluded from engagement processes. These considerations further informed development of the sustainability roadmap.

The creation of the sustainability roadmap (Table 1, step 5) [35] involved a non-systematic review of published and grey literature of models of roadmaps, building a roadmap, and the underpinning common theories for strategy and roadmap creation [41-56]. The roadmap architecture was built based on the principles of the Theory of Change model [52, 55-57] commonly used to focus the theoretical basis of a project, taking also into consideration the context in which the change will take place. Roadmaps consist of five elements that should be addressed [56, 58]:

i. Context of the initiative: analysis of current environment or landscape and actors who may influence the change.

ii. Long-term change: overall vision and desired longterm change and its expected benefits.

iii. Broad sequence of events/activities that may lead to long-term goal or change in given context.

iv. Assumptions: how change events/activities might happen and whether these activities and resulting outputs
PE driven by a strong signal from relevant authorities and by global harmonised guidelines

Regulators, health technology assessment (HTA) bodies, and payers requiring evidence of patient engagement for their decision-making and best practices supported by harmonised guidelines and methodologies issued by a global authoritative Consortium.

\section{PE one-stop approach/neutral hub}

$\mathrm{PE}$ is delivered by a neutral entity organising PE activities, promoting knowledge exchange and ensuring that funding is redistributed to build the capacity of patient organisations.

\section{PE reinforced by practice}

PE practices reinforced by a collective effort from all stakeholders who develop new ways and methods and learn from each other's experience. Metrics are crucial to identify and reinforce the most efficient practices.

\section{PE market approach}

PE is delivered by a mix of non-profit and for-profit organisations which could potentially derive financial benefit from their activities, reflecting existing practices in the current environment.
Fig. 2 Sustainability scenarios building blocks. These components were used to describe future scenarios with the greatest potential to accelerate the implementation of PE and make it sustainable. Each of these components can drive PE within stakeholder organisations and across the medicines development ecosystem 
are appropriate for influencing the desired change in given context.

v. Change diagram and narrative summary.

To populate this framework, further data were gathered (see Table 1, step 5) through stakeholder group consultations within the Consortium and informal interviews with regulators and other supra-national bodies with a potential role in harmonising PE requirements and setting priorities. The roadmap was further refined for relevance of roadmap elements (intermediate goals and actions) to the Central and Eastern Europe (CEE) region.

\section{The Patient Engagement Sustainability Roadmap}

The aim of a roadmap is to map out the desired future (not to predict it) and to provide a tool for collaborative strategic planning that enables stakeholders to make strategies and take actions towards that desired future [47].

\section{Context, Landscape and Barriers to PE}

Although opportunities for multi-stakeholder PE collaborations have become more common, the current landscape still presents barriers, including: cultural and political barriers; lack of knowledge and experience to carry out PE; methodological barriers; barriers to PE implementation; lack of human and financial resources; confidentiality and conflict of interest; and preconceptions about PE (Table 2). Such barriers have been described elsewhere [1, 38, 59-62].

Barriers vary according to country-level political, economic, socio-cultural, and technological contexts, e.g. some barriers are more challenging in CEE countries. For example, in some countries, socio-cultural differences in the perceived value and impact of PE by and between stakeholders (including governments and health ministries) and distrust are reported as important barriers hampering PE initiation or sustainability. Immature technological and financial infrastructure and support mechanisms for PO and PE related activities also contribute to sub-optimal PE. In general, PE and collaboration between stakeholders are more closely aligned and mature amongst Western EU Member States (MS) compared to CEE MS. Specific nuances of legal, ethical and cultural norms are often amplified across borders. Accordingly, some countries may need to address certain specific national barriers to PE before being able to tackle the wider range of goals and actions described in the roadmap (Box 1).

\section{End Goals}

The PE sustainability roadmap (33) outlined in this paper (Fig. 3) has the following elements: (a) the vision which defines 'why we need to take action', (b) the mission which describes 'what needs to be done', (c) the end goals, which are aspirational long-term outcomes, (d) the intermediate goals, which are major steps towards achieving the end goals, and (e) the actions needed to implement/deliver the goals and ultimately the vision.

The roadmap covers four end goals which must be met to overcome identified barriers and achieve meaningful and sustainable PE for better health outcomes:

1. Establish an ethical, trust-based collaboration amongst all PE stakeholders involved in medicines development.

2. Secure inclusive and diverse PE.

3. Embed PE in the mind-set, at every step and across organisations.

4. Ensure dedicated leadership and operational time, resources and funding for PE.

The actions described under each end goal are targeted to all organisations/institutions involved along the medicines development process.

\section{End Goal 1: Establish an Ethical, Trust-Based Collaboration Amongst all PE Stakeholders Involved in Medicines Development}

Trust is one of the critical underlying factors for sustained long-term collaboration and success. To achieve this goal, the three areas for action below were identified.

Include Patients as Equal Partners The patient community (patients, carers, advocates, POs) has a key role to play in increasing its credibility and recognition by other stakeholders. Strategic alignment across POs to bring a unified voice into decision-making bodies and policy strategy is critical in general and especially so across the CEE region, where there is often a more serious disconnect between POs and decision-makers. Community and partnership-building and knowledge transfer of best practices will help define PO strategy and objectives towards PE.

Achieving an equal partnership requires a strengthened organisational capacity for systematic strategic PE within all stakeholder organisations. Demonstrated commitment from executive leadership is needed to include patient insights in decision-making processes. In addition to developing their internal capacity, it is key that stakeholder organisations also contribute to building the capacity of the patient community to meaningfully engage and interact. Other actions required include raising awareness on PE impact and practices, 
Table 2 Barriers to Patient Engagement as Reported by Stakeholders

\begin{tabular}{|c|c|c|}
\hline Type & Description & Reported by ${ }^{\mathrm{a}}$ \\
\hline \multirow[t]{8}{*}{ Cultural, political } & $\begin{array}{l}\text { Language, cultural and political aspects of each country/ } \\
\text { region may make the adoption of PE practices difficult } \\
\text { (e.g. disease-related stigma may be more relevant in } \\
\text { some countries than others; changing priorities in } \\
\text { healthcare) }\end{array}$ & PO, HTA bodies \\
\hline & $\begin{array}{l}\text { Fragmentation (e.g. different diseases and geographic } \\
\text { regions, with different needs and interests, and compet- } \\
\text { ing for limited funding) }\end{array}$ & $\mathrm{PO}$ \\
\hline & $\begin{array}{l}\text { Lack of harmonised patient input in key policy develop- } \\
\text { ment areas due to competing priorities between POs }\end{array}$ & $\mathrm{PO}$ \\
\hline & English-centricity of PE practices & Regulators, HTA bodies \\
\hline & $\begin{array}{l}\text { Regulatory and legal environment not evolving along } \\
\text { with PE }\end{array}$ & Medicines developers \\
\hline & $\begin{array}{l}\text { PE practices may be designed with a pan-European } \\
\text { approach (e.g. engagement at EMA, global clinical } \\
\text { development programmes) }\end{array}$ & Multi-stakeholder CEE workshop \\
\hline & $\begin{array}{l}\text { Building and maintaining physical and virtual platforms } \\
\text { for discussion and exchange between organisations } \\
\text { challenging in some countries due competing strategies } \\
\text { of organisations }\end{array}$ & Multi-stakeholder CEE workshop \\
\hline & $\begin{array}{l}\text { In some countries, lack of PE and POs' visibility, and } \\
\text { lack of agreed communication channels between } \\
\text { patient organisations and other stakeholders PO can } \\
\text { have competing priorities as to where patient input is } \\
\text { focused }\end{array}$ & Multi-stakeholder CEE workshop \\
\hline \multirow[t]{8}{*}{$\begin{array}{l}\text { Lack of knowledge, skills or } \\
\text { experience }\end{array}$} & $\begin{array}{l}\text { Lack of PE skills and limited knowledge on how to } \\
\text { meaningfully involve patients in existing processes }\end{array}$ & $\begin{array}{l}\text { POs, medicines developers, regulators, HTA } \\
\text { bodies, ICH }\end{array}$ \\
\hline & $\begin{array}{l}\text { Lack of understanding of the public about their role } \\
\text { in medicines development and the role of regulatory } \\
\text { authorities }\end{array}$ & Regulators \\
\hline & Lack of PE at the early stages of a process/project & $\mathrm{PO}$ \\
\hline & Practices and processes not adapted to patients' needs & $\mathrm{PO}$ \\
\hline & $\begin{array}{l}\text { Lack of understanding of medical discussions and deci- } \\
\text { sions }\end{array}$ & $\mathrm{PO}$ \\
\hline & Lack of patient leadership & $\mathrm{PO}$ \\
\hline & Lack of health and PE literacy & PO, medicines developers \\
\hline & $\begin{array}{l}\text { Lack of knowledge on how to identify the right patients } \\
\text { for the required activity }\end{array}$ & Medicines developers, HTA bodies \\
\hline \multirow[t]{7}{*}{ Methodological } & Patients' needs not present & $\mathrm{PO}$ \\
\hline & Superficial engagement with HTA bodies and payers & $\mathrm{PO}$ \\
\hline & $\begin{array}{l}\text { Lack of knowledge on how to apply methodologies to } \\
\text { capture and use patients' insights }\end{array}$ & Medicines developers \\
\hline & $\begin{array}{l}\text { Existing evidence to prove that PE leads to better health } \\
\text { outcomes is still immature }\end{array}$ & Medicines developers \\
\hline & $\begin{array}{l}\text { Insufficient data to demonstrate value and impact of PE } \\
\text { for all stakeholders and that ultimately it may not be } \\
\text { perceived as a priority to be addressed }\end{array}$ & PO, medicines developers \\
\hline & $\begin{array}{l}\text { Lack of alignment across authorities on how to define } \\
\text { and integrate a PE framework that is applicable to the } \\
\text { local population needs and policies }\end{array}$ & Multi-stakeholder CEE workshop \\
\hline & $\begin{array}{l}\text { Little experience integrating and weighting patients' data } \\
\text { vs clinical data }\end{array}$ & Regulators \\
\hline \multirow[t]{2}{*}{ Implementation of PE } & $\begin{array}{l}\text { Lack of accountability mechanism of patient engagement } \\
\text { and of defined policies and practices }\end{array}$ & Medicines developers \\
\hline & Lack of harmonised approach to PE & Medicines developers \\
\hline
\end{tabular}


Table 2 (continued)

\begin{tabular}{|c|c|c|}
\hline Type & Description & Reported by ${ }^{\mathrm{a}}$ \\
\hline & $\begin{array}{l}\text { Prioritisation of PE activities where the patients' voice } \\
\text { adds more value vs including patients' voice in all } \\
\text { activities }\end{array}$ & Regulators \\
\hline & Practical aspects and logistics & Regulators \\
\hline & $\begin{array}{l}\text { EU guidelines and frameworks not transferable at local } \\
\text { level }\end{array}$ & $\mathrm{PO}$ \\
\hline \multirow[t]{9}{*}{ Lack of resources } & $\begin{array}{l}\text { Lack of appropriate culture and human and financial } \\
\text { resources at organisational level }\end{array}$ & $\begin{array}{l}\text { POs, medicines developers, regulators, HTA } \\
\text { bodies }\end{array}$ \\
\hline & $\begin{array}{l}\text { Lack of an organisational culture supportive of PE } \\
\text { amongst upper management }\end{array}$ & Medicines developers \\
\hline & $\begin{array}{l}\text { Long-term efforts and relationships required for optimal } \\
\text { patient engagement outcomes might not align with the } \\
\text { tight timelines of medicines development, hence risk- } \\
\text { ing patient engagement sustainability }\end{array}$ & Medicines developers \\
\hline & $\begin{array}{l}\text { Lack of financial resources to cover the expenses } \\
\text { incurred }\end{array}$ & $\mathrm{PO}$ \\
\hline & $\begin{array}{l}\text { Funding limited to short-term projects and not ensuring } \\
\text { long-term sustainability }\end{array}$ & PO, multi-stakeholder CEE workshop \\
\hline & Lack of funding diversification & PO, multi-stakeholder CEE workshop \\
\hline & $\begin{array}{l}\text { The lack of continuity of patient representatives (due to } \\
\text { disease burden or low patient numbers, such as in rare } \\
\text { or complex diseases) results in loss of knowledge and } \\
\text { expertise and limit the availability human resources }\end{array}$ & $\mathrm{PO}$ \\
\hline & $\begin{array}{l}\text { Lack of funding to support development of new patient } \\
\text { advocates and leaders for long-term activism }\end{array}$ & $\mathrm{PO}$ \\
\hline & $\begin{array}{l}\text { Lack of organisational capacity may prevent from incor- } \\
\text { porating good practices }\end{array}$ & PO, medicines developers, others \\
\hline \multirow[t]{4}{*}{$\begin{array}{l}\text { Conflict of interest and confiden- } \\
\text { tiality }\end{array}$} & $\begin{array}{l}\text { Lack of public (government/health ministry) funding for } \\
\text { PO }\end{array}$ & PO, multi-stakeholder CEE workshop \\
\hline & Funding coming from a single source (private funding) & PO, multi-stakeholder CEE workshop \\
\hline & $\begin{array}{l}\text { Risk of patients' losing their independence due to profes- } \\
\text { sionalization (e.g. becoming consultants) }\end{array}$ & Regulators \\
\hline & $\begin{array}{l}\text { Confidentiality barrier makes for lack of transparency } \\
\text { and low PE }\end{array}$ & Regulators \\
\hline \multirow[t]{5}{*}{ Preconceptions } & $\begin{array}{l}\text { Preconceptions about the value of the contribution of } \\
\text { some patient groups (such as children and young } \\
\text { patients and people living with dementia) and the chal- } \\
\text { lenges of involving them }\end{array}$ & POs \\
\hline & $\begin{array}{l}\text { In some CEE countries there may be a perception of a } \\
\text { lack of value of any contribution of patients or patient } \\
\text { organisations }\end{array}$ & $\mathrm{PO}$ \\
\hline & Lack of understanding of the value of PE & $\mathrm{PO}$ \\
\hline & Initial mistrust from engaging stakeholders & $\mathrm{PO}$ \\
\hline & Negative perception of industry engaging with patients & Medicines developers \\
\hline
\end{tabular}

${ }^{a}$ Sources Informal interviews with regulators (European Medicines Agency, UK Medicines and Healthcare Products Regulatory Agency, International Coalition of Medicines Regulatory Authorities) and the International Council for Harmonisation of Technical Requirements for Pharmaceuticals for Human Use (ICH). Internal consultations with PARADIGM Consortium patient organisations (PO) partners; PARADIGM industry partners (medicines developers). Multi-stakeholder CEE workshop on patient engagement (see Table 1 for further details)

listening to advocacy campaigns from initiatives promoting $\mathrm{PE}$ and building alliances with institutions that already work with established processes of PE.
Identify the Right Incentives and Motivators for PE for All Stakeholders Using a monitoring and evaluation framework to measure and demonstrate the impact of PE activities can help build the motivation, incentives and subsequent cultural shift to improve PE uptake in stakeholder 
Box 1. Priority activities for PE sustainability in the CEE region

- Harmonise the patient voice throughout the official governmental and health ministry channels. Include expertise of academia and health care professionals (HCP).

- Align mission and strategies across patient organisations to maximise PE impact with limited resources.

- Increase education, health literacy and capabilities of patients and PO through bilateral exchange of knowledge, people and ideas.

- Diversify funding models for PO; fund for strategic long-term programmes and better leverage of existing partnerships with academic institutions, HCP bodies and cross border initiatives.

Box 1 Priority activities for PE sustainability in the CEE region

organisations $[4,5]$. Potential incentives could be internal to the organisation (e.g. a publicly recognised award given to staff/departments showing impact of their PE strategies and activities) or external (e.g. EURORDIS Black Pearl Awards, EFPIA Health Collaboration Award).

Improve the Societal Perception of Collaboration Between Patients, Their Organisations, and Other Stakeholders Collaborations between patients, their organisations and other stakeholders need to become standard practice. Hence all parties benefit from an improved external perception towards these collaborations which may also enhance the overall willingness to engage. Supportive actions include following an established, ethical and transparent framework to help deliver PE [63], communicating on successes and failures through established and trusted platforms, being involved in public-private partnerships, and ensuring that processes are ethical, transparent and relevant between PE and other activities to proactively manage and mitigate potential conflicts of interests.

\section{End Goal 2: Secure Inclusive and Diverse Patient Engagement}

Meaningfully Involve Patients and Their Representatives Patients' needs and perspectives may vary widely within and across diseases, geographic regions, sociodemographic and other characteristics. It is important for all parties to acknowledge this diversity and to develop practices that recognise, respect, and incorporate the heterogeneity of patient populations and perspectives. PE in medicines development calls for the best achievable balance between diversity and the expertise and experience required for the activity. Opportunities for involvement should be maximized to incorporate a full range of patient experiences and views.

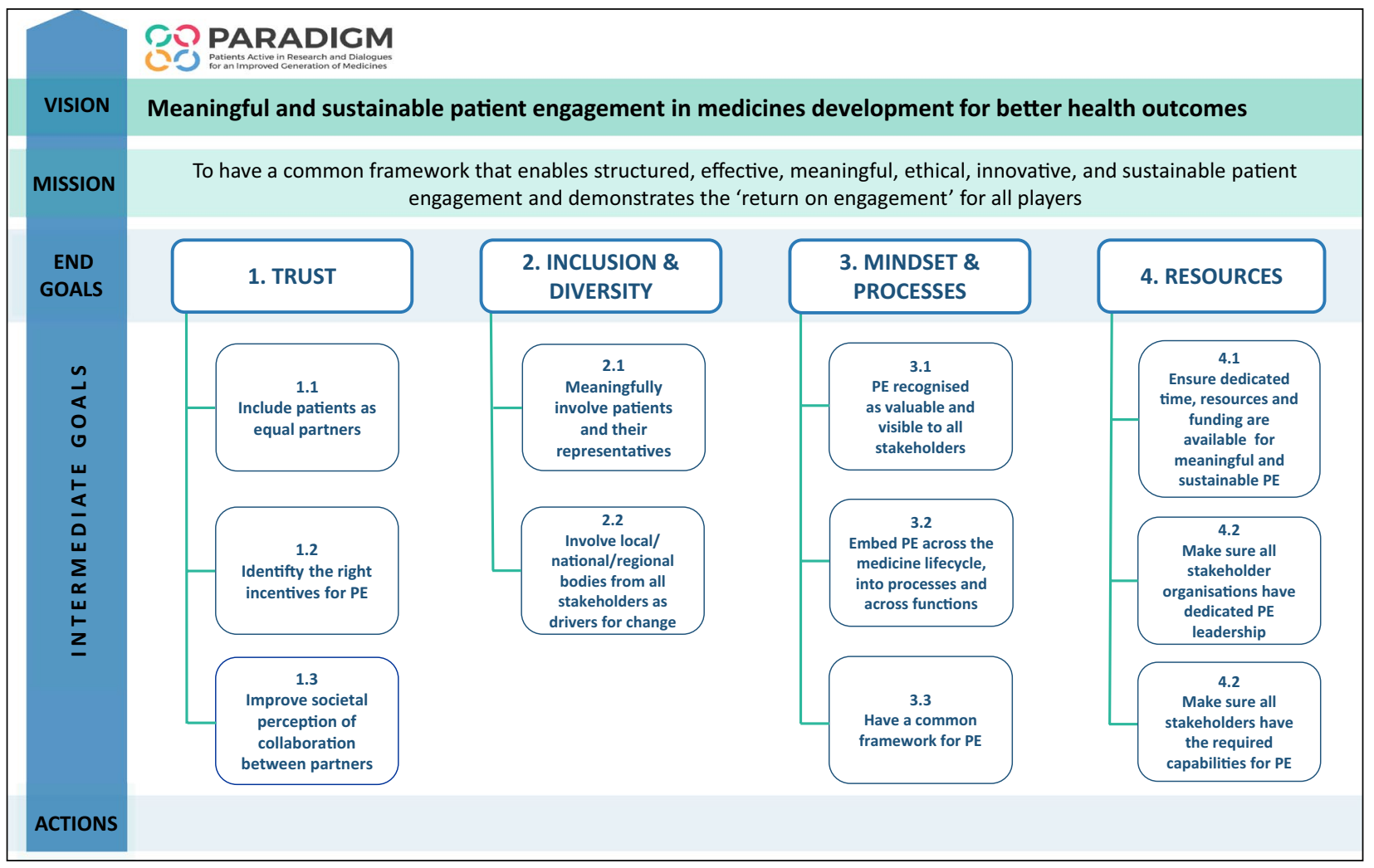

Fig. 3 PE sustainability roadmap visual summary 
Meaningful engagement could be fostered through publicly available established practices and mutually agreed objectives amongst the engaging parties where possible [64].

It is important to specifically consider requirements to engage with potentially vulnerable patient populations, patients with special needs, recently diagnosed patients and minority groups (seldom-heard patients). Involving potentially vulnerable populations is often considered a challenge and consequently these patients have historically largely been excluded from PE activities. Close relationships with POs linked to the condition experienced by these communities, or other organisations with expertise in how to involve the particularly vulnerable population, could enable their involvement in a meaningful way.

Organisations should raise awareness internally and externally, have policies in place on equality and diversity, as well as plan for the necessary resources, e.g. increased capacity to make the required time and accessibility adjustments and to tend to patient condition-specific needs. Integrated PE resources, guidance and tools, accompanied by training solutions (Fig. 4) provide the framework to implement ethical and meaningful PE; it will be crucial that these be disseminated to ensure widespread PE capacity-building across all parties. Capacity-building would include, but is

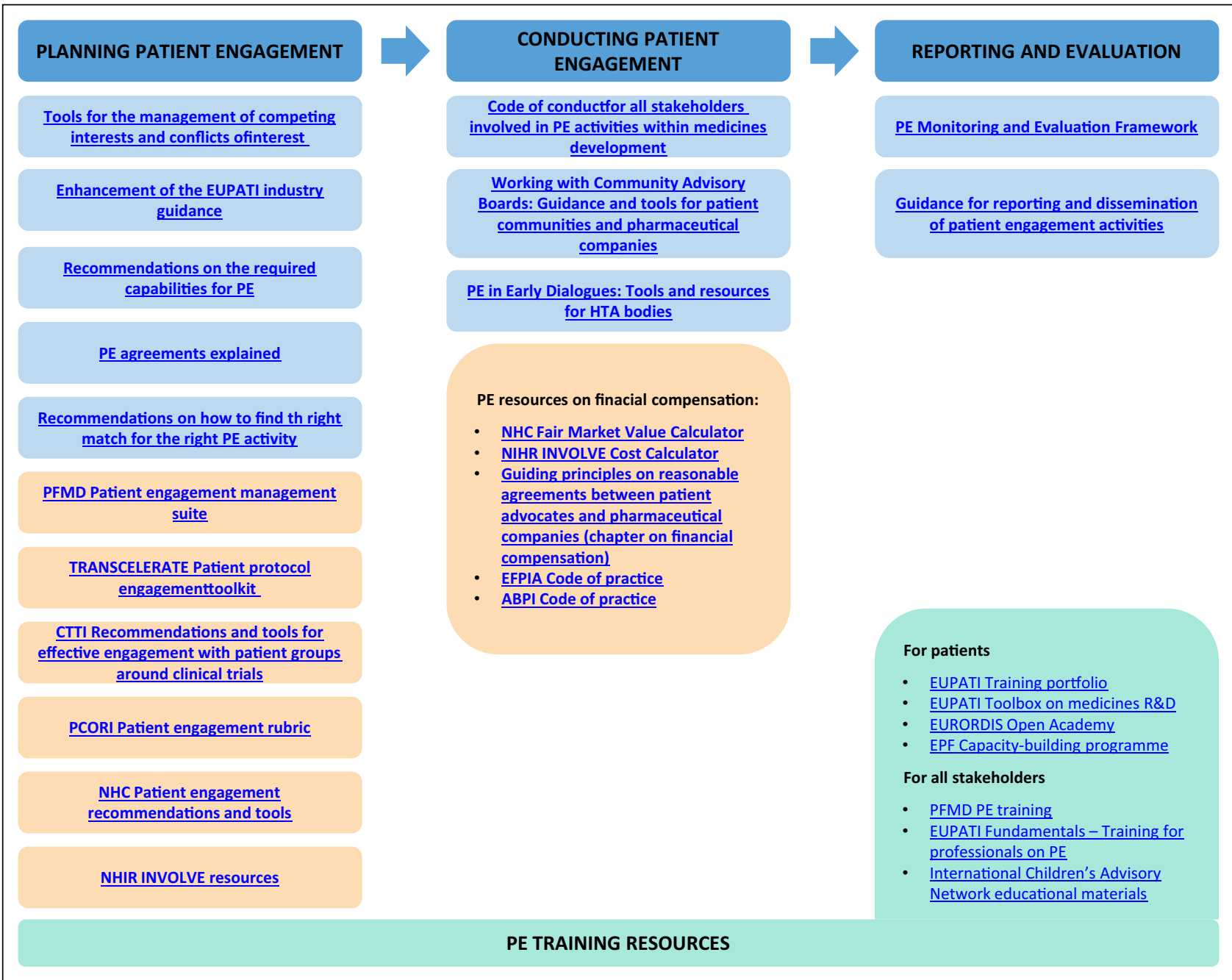

Fig. 4 Integrated resources, guidance and tools covering PE phases. List of existing PE frameworks to achieve sustainable PE. Top headings: Phases of patient engagement. All listed resources are available and free for use and links are available in the Supplementary information. Blue boxes: Recommendations, guidance and tools included in the PARADIGM Toolbox. Orange boxes: Tools and resources from existing patient engagement frameworks (non-exhaustive). Green boxes: PE training resources (non-exhaustive). All listed resources are available and free for use. $P E$ patient engagement, $P F M D$ patientfocused medicines development, CTTI Clinical Trials Transformation Initiative, PCORI Patient-Centered Outcomes Research Institute, NHC National Health Council, NIHR National Institute Health Research, EFPIA European Federation of Pharmaceutical Industries Association, ABPI Association of British Pharmaceutical Industry, EUPATI European Patients Academy on Therapeutic Innovation, EPF European Patients' Forum 
not restricted to, informal and formal mentorship and leadership training and programmes to ensure that knowledge and expertise are built and enhanced within the broad PE community. This is particularly important in the patient community as disease burden may limit their continuity or cancel their involvement. In order to focus activities in countries and regions needing more support, a regular benchmarking exercise is recommended to assess changing attitudes to the value of PE. All stakeholders can take such actions forward in their respective organisations to prioritise resources and drive change in the areas where it is most needed.

Involve Local/Regional/National Bodies from All Stakeholders as Drivers for Change PE often happens at a European or global level, e.g. European Medicines Agency (EMA), global clinical development programmes. However, local, regional and national stakeholder organisations have an important role to play as drivers for culture change in decentralised health systems. To optimise resource allocation, it is recommended to build on the existing strength of local/ regional/national bodies which should utilise networking platforms to share and adopt best practices, promoting knowledge exchange in a continuous and robust manner. This can facilitate active involvement in fora for discussion and/or decision-making. This ground level approach of PE, building cooperation, sharing techniques and adopting best practices must become widespread across geographies to ensure that strategies are considered in a broader context to achieve the best possible health outcomes.

\section{End Goal 3: Embed Patient Engagement in the Mind-set at Every Step and Across Organisations}

Recognize PE as Valuable and Visible to All Stakeholders Demonstrating and recognising the value that PE generates requires using different but complementary approaches. These include the generation of scientifically sound patient experience data through validated methodologies. This data should ideally be reported at regular intervals and analysed to provide evidence of the value added. Metrics are useful to identify how many and which type of insights provided during the PE activity have been implemented [5]. Ultimately, PE would lead to better health outcomes and therefore it is necessary to identify relevant indicators to reflect that patients' needs are met. Identifying negative outcomes and reverse engineering them is essential to understand where and what needs to be improved.

Other actions to increase value recognition of PE involve growing a community and/or health care ecosystem that can advocate for PE (both externally and internally) and identifying champions across stakeholder groups and organisations who can articulate the risk of not engaging patients within and outside their organisations. In addition, strategic partnership of POs with higher education institutions and learned societies will be key to train the future workforce of health care organisations, industry, regulatory authorities, HTA bodies and academia on the value of PE and the role of patients in medicines development and access. A similar approach has been described elsewhere for the co-production of patient-centred health care services [65]. Public research funders and policy-makers, as well as other public health organisations and authorities, and supra-national bodies can facilitate the creation of policies and guidelines to sustain PE.

\section{Embed PE Across the Medicines Lifecycle into Processes} and Functions Having a structured and consistent approach to PE processes across stakeholder organisations will help to reduce known barriers and sub-optimal PE practices. The course of actions will require that:

- Stakeholder organisations identify those points in the medicines development pathway where patients' insights are essential and would add most value (e.g. endpoint validation, development of patient-relevant outcome measures, benefit/risk assessment, etc.).

- Stakeholder organisations define and build the required capabilities and capacities to ensure implementation of PE; rely on champions to drive culture and organisational changes; continuously learn and share best practices; and monitor the adoption of existing practices, tools and recommendations.

- Regulatory authorities, HTA bodies and payers integrate the patients' voice into their procedures and develop a framework for engagement; set metrics to measure the degree of effectiveness of implementation of PE in their activities and processes and regularly report on their findings; ensure transparency; use existing platforms in regulatory science (Fig. 5) or other country/region-specific fora to enhance the PE discussion and ensure best practices.

Have a Common Regulatory Framework for PE Regulators and policy-makers may be expected to lead the way in setting a common framework for PE [66]. Public health authorities and other supra-national bodies may help establish clear priorities for $\mathrm{PE}$ as part of a broader public health strategy. On the other hand, harmonised methodologies are needed to help capture patients' insights in a systematic way that is accepted in regulatory (and HTA and payer) decisionmaking. The EMA Regulatory Science to 2025 has reflected on EMA's evolving approach to patient data, emphasising the use of methodologies to collect patient experience data for benefit/risk assessment, coordinating the approach to patient-reported outcomes (PROs) and promoting the use 


\begin{tabular}{|c|c|}
\hline \multirow{5}{*}{ Public-private consortia } & European Patients' Academy (EUPATI) \\
\hline & Patient-Focused Medicines Development (PFMD) \\
\hline & $\underline{\text { National Health Council (NHC) }}$ \\
\hline & Clinical Trials Transformation Initiative \\
\hline & Faster Cures \\
\hline \multirow[t]{2}{*}{ Harmonisation bodies } & $\begin{array}{l}\text { The International Council for Harmonisation of Technical Requirements for Pharmaceuticals for Human Use } \\
\text { (ICH) }\end{array}$ \\
\hline & Council for International Organizations of Medical Sciences (CIOMS) \\
\hline Supra-national bodies & World Health Organisation (WHO) \\
\hline \multirow{2}{*}{ Regulatory science platforms } & Heads of Medicines Agencies (HMA) network \\
\hline & International Coalition of Medicines Regulatory Authorities (ICMRA) \\
\hline \multirow{3}{*}{$\begin{array}{l}\text { Health Technology Assessment } \\
\text { and medicines pricing and } \\
\text { reimbursement networks }\end{array}$} & European network for Health Technology Assessment (EUnetHTA) \\
\hline & Health Technology Assessmentinternational (HTAi) \\
\hline & Medicine Evaluation Committee (MEDEV) \\
\hline \multirow{13}{*}{$\begin{array}{l}\text { Multi-stakeholder networking } \\
\text { platforms }\end{array}$} & Patient Engagement Open Forum (PEOF) \\
\hline & The European Federation of Pharmaceutical Industries and Associations (EFPIA) Annual Conference \\
\hline & European Medicines Agency Patients' and Consumers Working Party \\
\hline & European Federation of Pharmaceutical Industries and Associations (EFPIA) Patient Think Tank \\
\hline & European Patients Forum (EPF) Congress on Patient Involvement \\
\hline & European Conference on Rare Diseases (ECRD) \\
\hline & Health Technology Assessment International (HTAi) Annual Meeting \\
\hline & Drug Information Association (DIA) \\
\hline & $\begin{array}{l}\text { Biotechnology Innovation Organization } \\
\text { Partnering for cures (Faster Cures) }\end{array}$ \\
\hline & International Society for Pharmacoeconomics and Outcomes Research (ISPOR) Patient Representative \\
\hline & Roundtable \\
\hline & ISPOR conferences \\
\hline & $\overline{\text { PFMD Synapse for patient engagement }}$ \\
\hline
\end{tabular}

Fig. 5 Platforms important for PE sustainability. Links to all listed resources are available in the Supplementary Information

of core health-related quality of life PROs [67]. The US Food and Drug Administration (FDA) through the PatientFocused Drug Development (PFDD) programme [23] is developing four guidance documents for the collection and use of patient input to better inform product development and decision-making [20]. In addition, both EMA and FDA committed to formal collaboration, knowledge exchange and alignment of PE processes between the two agencies through different mechanisms [3]. In 2019, the Japanese Pharmaceuticals and Medical Devices Agency (PMDA) showed its commitment to PE by setting up a Patient Centricity Working Group to identify how patients could be involved in the Agency's activities and to develop guidance on PE at PMDA [68]. In addition, the International Council for Harmonisation of Technical Requirements for Pharmaceuticals for Human Use (ICH) could play a significant role in the harmonisation of PE practices in clinical trial programmes. ICH has started drafting a new version of ICH E8 General Considerations for Clinical Trials guideline, which includes general considerations on patient input into study design [69]. ICH will commit to ensure the upfront involvement of all parties in study planning [70]. The Council for International Organizations of Medical Sciences (CIOMS) created in 2018 the CIOMS Working Group XI on Patient Involvement and is now elaborating a future global guidance on patient involvement [71]. "Patient participation in the generation and utilization of safety and effectiveness data" will be one of the areas covered in this guidance. These concerted efforts will no doubt lead to more effective PE and generate solid evidence for regulatory, HTA and payer decision-making.

\section{End Goal 4: Ensure Dedicated Leadership and Operational Time, Resources and Funding for Patient Engagement}

Ensure Dedicated Time, Resources and Funding are Available for Meaningful and Sustainable PE Conducting optimal PE takes substantial resources, either in terms of operational time to carry out and deliver PE, increasing resources and augmenting staff competencies and departmental capacity, or securing continued funding for PE activities. Given the number and systematic activities proposed in the roadmap, meaningful and sustainable PE is unlikely to be achieved at current resource levels across stakeholder groups and organisations, hence human and financial resources will most likely need to be increased.

Although the industry is showing strong signs of progress in PE, the budgets, funding and resource allocation to PE units are not comparable to those dedicated for the engagement of other stakeholders (e.g. health care professionals and policy groups). There is a lack of funding for internal $P E$ activities and insufficient internal understanding of how 
grants, donations and sponsorships are allocated. Industry stakeholders must continue to elevate the discussion to the highest levels on the role and competencies required for PE.

Across Europe different financial models that support PE activities will likely be needed to ensure long-term sustainability of strategies and actions. Relevant financial resources in the CEE region are currently very limited and their use is mainly reactive which undermines sustainable responses. In the case of CEE POs, funding may come from a single source (e.g. private funding), resulting in a perception of lack of PO independence. In addition, such funding is usually aimed at short-term projects rather than strategic, longterm engagements that are designed to ensure stable and sustainable responses. This might be aggravated by funding diverted to other causes in the event of global health crises (see Limitations). Demonstrating the return on engagement may help to leverage more strategic funding.

Stakeholder organisations should assess their needs for the time, personnel and funding required to conduct meaningful PE and ensure that they are addressed where it is most relevant and/or likely to be of significant impact and benefit. Depending on the type of stakeholder, other actions could involve seeking alternative funding models through national and cross-border initiatives that can promote $\mathrm{PE}$ processes and practices. A preferred model is one by which the financial independence of POs is secured. There can be no meaningful $\mathrm{PE}$ if the implementation of a compensation framework for patient participants undermines patient community participation or results in feelings of disrespect or mistrust. It is therefore critical that compensation is paid according to fair market value standards and compliant with local laws and regulations. It is also required to ensure that participation in PE activities does not place a financial burden on patients.

\section{Make Sure All Stakeholder Organisations have Dedicated PE Leadership and the Required Capabilities Regarding human resources, it is crucial to ensure dedicated PE lead- ers who can drive culture and organisational changes and also to equip the organisations with functions holding the required core competencies, processes, tools and systems to implement PE [72].}

\section{Resources and Tools for Sustainability}

The Consortium developed a toolbox [73] (Fig. 4) following a process of needs assessment, gap analysis and co-production to increase stakeholders' preparedness for PE. The toolbox was not created in a vacuum but built on the back of global initiatives (e.g. TransCelerate, CTTI, NHC, PCORI, PFMD, EUPATI) and the experience of Consortium partners, and provides guidance and practical recommendations along the different phases of PE. Planning for sustainability involves knowing the available tools and using them at the right stages to ensure consistent PE practices. The implementation of the actions proposed in the roadmap will be facilitated using such tools and will result in enhancement and optimization of existing processes within organisations. The organisations within the PARADIGM Consortium are already implementing this toolbox in their activities and are highly committed to disseminate it and encourage their use within their respective constituencies and beyond. In addition, the Patient-Focused Medicines Development (PFMD) Consortium and the EUPATI Initiative will host the PARADIGM toolbox in their respective sites hence ensuring that PARADIGM outputs are widely available to stakeholder organisations beyond the Consortium [74].

Sharing the knowledge and experiences of doing PE, reaching out to stakeholders for alignment and validation, and assessing the level of institutionalisation of PE practices are essential mechanisms of a system-wide strategy to sustain PE in medicines development. Evaluation ensures alignment of the activities with the proposed framework and enables the making of necessary adaptations to reflect new knowledge and insights. Figure 5 features established public-private consortia that promote, support and develop tools for PE; harmonisation and supra-national bodies with a potential role in harmonising PE requirements and setting priorities; regulatory science, HTA bodies and payers' networks that could enhance the PE discussion around the requirement of patient experience data in their decisionmaking; and multi-stakeholder networking platforms where stakeholder alignment and trust-building, together with cross-pollination of experiences and best practices, take place.

\section{Role of Stakeholder Organisations}

The change process towards sustained PE is expected to occur at individual, organisational and systemic levels, and may happen at a different pace across geographies and organisations. The roadmap does not propose a linear timeline for actions to unfold, but instead actions can occur at non-linear checkpoints as described elsewhere [48]. This non-linear approach proposes that in the short term, organisational strategy may be driven by tactics, which are underpinned by available data, evidence and certainty. Moving beyond this to the mid- and long-term, organisations must accept some uncertainty as they continuously reflect on their learnings and recalibrate their strategy and tactics to their new starting point for systems level evolution.

When confronted with mixed urgent and important priorities (e.g. setting up collaborations to develop treatments for COVID-19 vs setting up an organisation-wide PE training), organisations risk falling into the 'mere-urgency trap' and focus efforts in short-term activities that may prevent them 
from fulfilling long-term goals [22]. Whilst the importance of patient-centeredness and PE is now beyond question for most stakeholders, achieving the roadmap's vision will require reassessing the priority given to $\mathrm{PE}$.

The implementation of the actions described in the roadmap is underpinned by two fundamental mechanisms: (1) the use of integrated PE resources and toolkits that can help all stakeholders to manage existing experiences and resources and optimize them, i.e., "to not reinvent the wheel" (Fig. 4); and (2) knowledge sharing and benchmarking within and between organisations (Fig. 5). It is essential that organisations encourage culture and process change through education and knowledge sharing of best practices internally and externally and develop the competency to measure and report on the impact of PE, including risks and impact of not doing it. External engagement will be crucial in helping to drive internal change.

The roadmap architecture and assumptions have been built based on the principles of the Theory of Change to propose a set of actions, the connections between them and the desired long-term change (i.e., end goals and vision) [58]. Monitoring progress of the implementation of the actions is necessary to redefine or update the strategy according to the evidence gathered at given checkpoints. Each stakeholder organisation may already be measuring implementation of PE practices internally and could follow similar, albeit different, methods to monitor roadmap adoption. Collective responsibility and collaborations within and across stakeholder groups and organisations will be crucial to drive the actions forward. Benchmarking mechanisms could take many forms such as open dialogues and multilateral exchanges of knowledge, ideas and good practices. External engagement through multi-stakeholder platforms will be key to monitor if and how changes are implemented across organisations and stakeholder groups. The roadmap encourages stakeholders to make the most of existing initiatives and networking platforms for knowledge diffusion and experience exchange, but also as mechanisms to benchmark progress towards the vision (Fig. 5). The emergence of the IMI-PARADIGM Patient Engagement Open Forum (PEOF) [75], with its hands-on approach to advance PE, could also strengthen multi-stakeholder collaborations and the collective evolution of the PE community. Former PEOF organisers (i.e. EUPATI, PFMD) together with the European Patients' Forum reiterated their commitment and joined efforts to continue organising future editions of the PEOF following PARADIGM recommendations [74].

\section{Limitations}

The roadmap is intended to be aspirational and envisions that PE in medicines development is sustainable, i.e. that $\mathrm{PE}$ as 'business as usual' is achievable. Despite a growing shared culture, stakeholders hold different expectations and objectives regarding PE in medicines development and therefore might have different attitudes towards the need for and impact of change [66]. We acknowledge that top-down strategies (e.g. regulators requiring evidence of $\mathrm{PE}$ as part of a medicine registration dossier) may help accelerate change (e.g. patient input in clinical programme).

Although the actions are intended to be taken forward by all stakeholders involved, it is acknowledged that there is no single entity in Europe responsible for implementing or updating the roadmap. As a result, there will be a need to adjust the roadmap depending on differing and possibly competing remits, processes or other influences. In some countries, it will be important to address existing barriers before its implementation (Box 1). Since periodic benchmarking of progress is currently done separately by each stakeholder using different mechanisms, it will not be easy to arrive at realistic and shared benchmarks for the roadmap across Europe. Informal collaborations amongst stakeholder groups through knowledge-sharing platforms and other mechanisms focused on PE will play a role in advancing the strategy (Fig. 5).

The roadmap has not been stress tested within any entity or stakeholder to identify possible impediments to its implementation. Soft power is relied upon to implement the roadmap and thus some elements may not be practical or feasible to implement by one or more stakeholders at any given step. The assumptions that underpin the current PE landscape and desired future state have a degree of uncertainty and limited accuracy, and therefore the roadmap may well require updating in the mid-to long-term.

The roadmap might be affected by global health crises which have an impact on countries' political stability and the availability of resources in health care systems. PE may be particularly reduced in such circumstances, as observed during the COVID-19 pandemic [76]. POs have experienced operational difficulties and shifting priorities due to the increased demand to support their community [77], which has led to reducing other activities such as fundraising or involvement in PE activities. Nevertheless, public health emergencies may also bring opportunities for increased collaboration in which patient- and citizen-driven change may become more relevant [76-78]. The expansion of virtual events and platforms during the pandemic has increased patients' accessibility to these events, thus facilitating discussion and exchange between patients and other stakeholders in an unprecedented manner. 


\section{Conclusion: Call to Action}

Benchmarking progress and evolving strategies are not the final steps; sustaining change requires a process of continued learning and improvement, experience sharing and production of more change (if needed) [52]. Stakeholders have now a roadmap that can be adopted and adapted within their own organisations depending on the maturity of their own PE practices. The roadmap recommends taking a series of actions towards four key end goals to ensure that PE is sustained through (1) mutual trust across stakeholder groups; (2) inclusive and diverse PE, with the involvement of organisations at all levels (not only central/global); (3) culture drivers to embed PE within and across organisations; and (4) dedicated human and financial resources. This roadmap can be regarded as a succession of interconnected milestones that are achievable thanks to the existing resources. IMI-PARADIGM has delivered a robust framework, that in synergy with others (Fig. 4), will help implementing PE processes and consolidating existing ones within individual organisations, thus fostering PE and making it sustainable. PARADIGM has also contributed to strengthen organisations' PE readiness not only by providing actionable tools, but by bringing stakeholders together and breaking down fragmentation in the medicines development ecosystem.

However, widespread adoption and implementation of the roadmap relies on a PE community open to honest collaboration and exchange beyond their own organisation and stakeholder group to sustain change across the medicines development ecosystem. Now more than ever, sustainable PE seems within reach if all stakeholders take joint and determined action.

\section{Box 2. Call to action}

Patients and patient organisations (POs), medicines developers, academia, regulatory authorities, HTA bodies, payers, policy makers and public research funders are called to:

- Identify, develop and share good practices that are replicable and transferable within and between stakeholder groups;

- Disseminate best practices across all stakeholder groups and health systems;

- Build the conditions for resilient and sustainable PE;

- Develop further evidence of improved performance and health outcomes through PE; and

- Foster a sustainable PE ecosystem that increases engagement opportunities and the resources to expand patients' engagement capacity.

Box 2 Call to action to the PE community

\section{Acknowledgements}

We want to thank Virginie Hivert, Concha Mayo and Zachary Hallinan for their significant contributions to the development of the patient engagement sustainability roadmap, and to Daniel O'Connor, Colin Attrill, Mike Dykes, Maria Mavris, Nathalie Bere, Lenita LindströmGommers, Andrea Furia-Helms and Agnès Saint-Raymond for their time and insights during the interviews conducted to populate the roadmap. In addition, we would like to thank all PARADIGM work package 6 partners for their engagement and support throughout the development of the sustainability strategy.

\section{Author Contributions}

$\mathrm{SDF}, \mathrm{BT}, \mathrm{MB}, \mathrm{EM}, \mathrm{MJV}, \mathrm{NBe}, \mathrm{VP}, \mathrm{KR}$ and $\mathrm{EF}$ were involved in the conception and design phase of the roadmap. MCB, SDF, BT, MB, $\mathrm{EM}, \mathrm{KR}$ and $\mathrm{EF}$ were involved in the conduct of interviews, consultations and/or workshops. MCB, SDF, BT, MB, EM, NBr, LMcK, JS, MJV, NBe, JGB, VP, KR and EF were involved in the development of the sustainability roadmap contents and in the writing of the manuscript.

\section{Funding}

PARADIGM has received funding from the Innovative Medicines Initiative 2 Joint Undertaking under Grant Agreement 777450. This Joint Undertaking receives support from the European Union's Horizon 2020 Research and Innovation Programme and EFPIA.

\section{Declarations}

\section{Conflict of interest}

The following authors have no conflict of interest to disclose: Maria Cavaller Bellaubi, Stuart D. Faulkner, Bryan Teixeira, Mathieu Boudes, Eva Molero, Nicholas Brooke, Laura McKeaveney, Jeffrey Southerton, Maria José Vicente, Juan García-Burgos, Vinciane Pirard, Kirsty Reid and Elisa Ferrer. Neil Bertelsen works with a variety of stakeholder groups on patient involvement in healthcare decisions. This includes work as a consultant to patient groups and to the industry.

\section{Open Access}

This article is licensed under a Creative Commons Attribution 4.0 International License, which permits use, sharing, adaptation, distribution and reproduction in any medium or format, as long as you give appropriate credit to the original author(s) and the source, provide a link to the Creative Commons licence, and indicate if changes were made. The images or other third party material in this article are included in the article's Creative Commons licence, unless indicated otherwise in a credit line to the material. If material is not included in the article's Creative Commons licence and your intended use is not permitted by statutory regulation or exceeds the permitted use, you will need to obtain permission directly from the copyright holder. To view a copy of this licence, visit http://creativecommons.org/licenses/by/4.0/.

\section{Supplementary Information}

The online version contains supplementary material available at https:// doi.org/10.1007/s43441-021-00282-z. 


\section{References}

1. Hoos A, Anderson J, Boutin M, et al. Partnering with patients in the development and lifecycle of medicines: a call for action. Ther Innov Regul Sci. 2015;49(6):929-39. https://doi.org/10. $1177 / 2168479015580384$.

2. Getz K. Reflections on the evolution of patient engagement in drug development. Pharm Med. 2019;33(3):179-85. https://doi.org/10. 1007/s40290-019-00284-1.

3. Mavris M, Helms AF, Bere N. Engaging patients in medicines regulation: a tale of two agencies. Nat Rev Drug Discov. 2019. https://doi.org/10.1038/d41573-019-00164-y.

4. Vat LE, Finlay T, Jan Schuitmaker Warnaar T, et al. Evaluating the "return on patient engagement initiatives" in medicines research and development: a literature review. Health Expect. 2019;23(1):5-18. https://doi.org/10.1111/hex.12951.

5. Vat LE, Finlay T, Robinson P, et al. Evaluation of patient engagement in medicines development: a multi-stakeholder framework with metrics. Health Expect. 2021. https://doi.org/10.1111/hex. 13191.

6. Geissler J, Ryll B, di Priolo SL, Uhlenhopp M. Improving patient involvement in medicines research and development: a practical roadmap. Ther Innov Regul Sci. 2017;51(5):612-9. https://doi. org/10.1177/2168479017706405.

7. Manafò E, Petermann L, Vandall-Walker V, Mason-Lai P. Patient and public engagement in priority setting: a systematic rapid review of the literature. PLoS ONE. 2018;13(3):e0193579-18. https://doi.org/10.1371/journal.pone.0193579.

8. Sheridan S, Schrandt S, Forsythe L, Hilliard TS, Paez KA. The PCORI engagement rubric: promising practices for partnering in research. Ann Fam Med. 2017;15(2):165-70. https://doi.org/10. 1370/afm.2042.

9. Bloom D, Beetsch J, Harker M, et al. The rules of engagement: CTTI recommendations for successful collaborations between sponsors and patient groups around clinical trials. Ther Innov Regul Sci. 2018;52(2):206-13. https://doi.org/10.1177/21684 79017720247

10. European AIDS Treatment Group (EATG). The European Community Advisory Board (ECAB). http://www.eatg.org/ecab/. Accessed 31 Jan 2021.

11. EURORDIS-Rare Diseases Europe. Community Advisory Board Programme (EUROCAB). https://www.eurordis.org/content/euror dis-community-advisory-board-cab-programme. Accessed 31 Jan 2021.

12. Revised framework for interaction between the European Medicines Agency and patients and consumers and their organisations. https://www.ema.europa.eu/en/documents/other/revised-frame work-interaction-between-european-medicines-agency-healt hcare-professionals-their_en.pdf. Published 16 December 2016. Accessed 31 Jan 2021.

13. Parallel consultation with regulators and health technology assessment bodies. European Medicines Agency (EMA) and European Network for Health Technology Assessment (EUnetHTA). https:// www.ema.europa.eu/en/human-regulatory/research-development/ scientific-advice-protocol-assistance/parallel-consultation-regul ators-health-technology-assessment-bodies. Accessed 31 Jan 2021.

14. Mechanisms of coordinated access to orphan medicinal products (MoCA). Revised terms of reference. https://www.eurordis.org/ content/moca. Published 25 January 2016. Accessed 31 Jan 2021.

15. Outcome report on pilot to involve patients in benefit/risk discussions at CHMP meetings. https://www.ema.europa.eu/en/docum ents/report/outcome-report-pilot-involve-patients-benefit/riskdiscussions-chmp-meetings_en.pdf. Published 3 March 2017. Accessed 31 Jan 2021.
16. UK Medicines and Healthcare Products Regulatory Agency (MHRA) guidance: opportunities for patients and the public to be involved in the work of the MHRA. https://www.gov.uk/guida nce/opportunities-for-patients-and-the-public-to-be-involved-inthe-work-of-the-mhra. Accessed 31 Jan 2021.

17. European Network for Health Technology Assessment (EUnetHTA). Patient input in Relative Effectiveness Assessments. https://eunethta.eu/wp-content/uploads/2019/06/Final_290519_ Patient-Input-in-REAs.pdf. Published May 29, 2019. Accessed 31 Jan 2021.

18. Berglas S, Jutai L, MacKean G, Weeks L. Patients' perspectives can be integrated in health technology assessments: an exploratory analysis of CADTH Common Drug Review. Res Involv Engagem. 2016;2(1):21-13. https://doi.org/10.1186/s40900-016-0036-9.

19. Leopold $\mathrm{C}, \mathrm{Lu} \mathrm{CY}$, Wagner AK. Integrating public preferences into national reimbursement decisions: a descriptive comparison of approaches in Belgium and New Zealand. BMC Health Serv Res. 2020. https://doi.org/10.1186/s12913-020-05152-2.

20. FDA patient-focused drug development guidance series for enhancing the incorporation of the patient's voice in medical product development and regulatory decision making. https://www. fda.gov/drugs/development-approval-process-drugs/fda-patientfocused-drug-development-guidance-series-enhancing-incorporat ion-patients-voice-medical. Accessed 31 Jan 2021.

21. Rogers EM. Diffusion of innovations, 5th edn. https://books. google.es/books?id=9U1 K5LjUOwEC\&printsec $=$ frontcover\& redir_esc $=\mathrm{y} \# \mathrm{v}=$ onepage $\& \mathrm{q} \& \mathrm{f}=$ false.

22. Scroggs L. The Eisenhower matrix. https://todoist.com/es/produ ctivity-methods/eisenhower-matrix. Accessed 31 Jan 2021.

23. Perfetto EM, Burke L, Oehrlein EM, Epstein RS. Patient-focused drug development: a new direction for collaboration. Med Care. 2015;53(1):9-17. https://doi.org/10.1097/MLR.0000000000 000273.

24. Hunter A, Facey K, Thomas V, et al. EUPATI guidance for patient involvement in medicines research and development: health technology assessment. Front Med. 2018;5:334-410. https://doi.org/ 10.3389/fmed.2018.00231.

25. Klingmann I, Heckenberg A, Warner K, et al. EUPATI and patients in medicines research and development: guidance for patient involvement in ethical review of clinical trials. Front Med. 2018;5:230-311. https://doi.org/10.3389/fmed.2018.00251.

26. Haerry D, Landgraf C, Warner K, et al. EUPATI and patients in medicines research and development: guidance for patient involvement in regulatory processes. Front Med. 2018;5:74-11. https:// doi.org/10.3389/fmed.2018.00230.

27. Warner K, See W, Haerry D, Klingmann I, Hunter A, May M. EUPATI guidance for patient involvement in medicines research and development $(\mathrm{R} \& \mathrm{D})$; guidance for pharmaceutical industryled medicines R\&D. Front Med. 2018;5:929-38. https://doi.org/ 10.3389/fmed.2018.00270.

28. Elmer M, Florek C, Gabryelski L, et al. Amplifying the voice of the patient in clinical research: development of toolkits for use in designing and conducting patient-centered clinical studies. Ther Innov Regul Sci. 2020. https://doi.org/10.1007/ s43441-020-00176-6.

29. Deane K, Delbecque L, Gorbenko O, et al. Co-creation of patient engagement quality guidance for medicines development: an international multistakeholder initiative. BMJ Innov. 2019;5(1):43-55. https://doi.org/10.1136/bmjinnov-2018-000317.

30. Hickey G, Brearley S, Coldham T, et al. NIHR Guidance on coproducing a research project. In: INVOLVE. 2018, Southampton. https://www.invo.org.uk/wp-content/uploads/2019/04/Copro_ Guidance_Feb19.pdf. Accessed 31 Jan 2021.

31. Abma TA, Pittens CACM, Visse M, Elberse JE, Broerse JEW. Patient involvement in research programming and implementation: a responsive evaluation of the Dialogue Model for research 
agenda setting. Health Expect. 2015;18(6):2449-64. https://doi. org/10.1111/hex.12213.

32. Supple D, et al. From tokenism to meaningful engagement: best practices in patient involvement in an EU project. Res Involv Engagem. 2015. https://doi.org/10.1186/s40900-015-0004-9.

33. Price A, Albarqouni L, Kirkpatrick J, et al. Patient and public involvement in the design of clinical trials: an overview of systematic reviews. J Eval Clin Pract. 2017;24(1):240-53. https://doi. org/10.1111/jep.12805.

34. Innovative Medicines Initiative (IMI) PARADIGM (Patients Active in Research And Dialogues for an Improved Generation of Medicines). https://imi-paradigm.eu. Accessed 31 Jan 2021.

35. Deliverable D6.5. Patient Engagement Sustainability Roadmap. PARADIGM WP6 Sustainability Strategy. https://imi-paradigm. eu/wp-content/uploads/2020/09/PARADIGM-D6.5_PE-Susta inability-roadmap_FINAL.pdf. Accessed 1 Feb 2021.

36. Boutin M, Dewulf L, Hoos A, et al. Culture and process change as a priority for patient engagement in medicines development. Ther Innov Regul Sci. 2017;51(1):29-38. https://doi.org/10.1177/ 2168479016659104

37. Deliverable D6.1 Assessment review of existing sustainability models. PARADIGM WP6 Sustainability Strategy. https://imiparadigm.eu/wp-content/uploads/2019/11/M09_D-6.1-Asses sment-review-of-the-existing-sustainability-models-1.pdf. Published November 2018. Accessed 1 Feb 2021.

38. Faulkner SD, Li SS, Pakarinen C, et al. Understanding multistakeholder needs, preferences and expectations to define effective practices and processes of patient engagement in medicine development: a mixed-methods study. Health Expect. 2021. https://doi. org/10.1111/hex.13207.

39. Deliverable D6.2. List of the relevant models addressing sustainability for all stakeholders. PARADIGM WP6 Sustainability Strategy. https://imi-paradigm.eu/wp-content/uploads/2019/11/ M16_D-6.2-Shortlist-of-sustainability-scenarios-V1.3_FINAL_ Submission_IMI-1.pdf. Published May 7, 2018. Accessed 1 Feb 2021.

40. Deliverable D6.3. Refined list of the relevant models addressing sustainability for all stakeholders. PARADIGM WP6 Sustainability Strategy. https://imi-paradigm.eu/wp-content/uploads/2020/09/ M22_D6.3-Refined-list-of-the-relevant-models-addressing-susta inability-for-all-stakeholders.pdf. Accessed 1 Feb 2021.

41. The Association of the British Pharmaceutical Industry (ABPI) medicines optimisation 2016-2020. https://abpi.org.uk/media/ 1668/abpi_slideck_chapter5.pdf. Accessed 31 Jan 2021.

42. Government of Canada SME suitability roadmap 2012. https:// www.ic.gc.ca/eic/site/csr-rse.nsf/eng/h_rs00174.html\#roadmap. Accessed 31 Jan 2021.

43. Cundall Sustainability Roadmap. https://cundall.com/Knowledgeh ub/Sustainability-Roadmap.aspx. Accessed 31 Jan 2021.

44. European Medicines Agency (EMA) Heads of Medicines Agencies (HMA)-EMA Joint Big Data Taskforce-summary report 2019. https://www.ema.europa.eu/en/documents/minutes/hma/ ema-joint-task-force-big-data-summary-report_en.pdf. Accessed 31 Jan 2021.

45. The Economist. Scenario planning. https://www.economist.com/ news/2008/09/01/scenario-planning. Published 1 September 2008. Accessed 31 Jan 2021.

46. Strauss JD, Radnor M. Roadmapping for dynamic and uncertain environments. Res Technol Manag. 2016;47(2):51-8. https://doi. org/10.1080/08956308.2004.11671620.

47. Hasse JU, Weingaertner DE. From vision to action: roadmapping as a strategic method and tool to implement climate change adaptation - the example of the roadmap "water sensitive urban design 2020.” Water Sci Technol. 2016;73(9):2251-9. https://doi. org/10.2166/wst.2016.065.
48. Webb A. How to do strategic planning like a futurist. July 2019:18. https://hbr.org/2019/07/how-to-do-strategic-planning-like-afuturist. Accessed 31 Jan 2021.

49. Phaal R. Roadmapping. Cambridge roadmapping. https://www. cambridgeroadmapping.net/roadmapping. Accessed 31 Jan 2021.

50. Phaal R, Farrukh CJP, Probert DR. Technology roadmapping-a planning framework for evolution and revolution. Technol Forecast Soc Change. 2004;71(1-2):5-26. https://doi.org/10.1016/ S0040-1625(03)00072-6.

51. Ahmed MD, Sundaram D. Sustainability modelling and reporting: from roadmap to implementation. Decis Support Syst. 2012;53(3):611-24. https://doi.org/10.1016/j.dss.2012.02.004.

52. Ibrahim M, El-Zaart A, Adams C. Smart sustainable cities roadmap: readiness for transformation towards urban sustainability. Sustain Cities Soc. 2017. https://doi.org/10.1016/j.scs.2017.10. 008.

53. McMillan A. Roadmapping-agent of change. Res Technol Manag. 2016;46(2):40-7. https://doi.org/10.1080/08956308.2003. 11671553.

54. Kamtsiou V, Naeve A, Stergioulas LK, Koskinen T. Roadmapping as a knowledge creation process: the PROLEARN roadmap. J Univers Knowl Manag. 2006;1(3):163-73.

55. Innes JE, Booher DE. A turning point for planning theory? Overcoming dividing discourses. Plan Theory. 2013;14(2):195-213. https://doi.org/10.1177/1473095213519356.

56. Vogel I. ESPA guide to working with Theory of Change for research projects. https://www.imainternational.com/images/listi ng_doc/ESPA-Theory-of-Change-Manual-FINAL.pdf. Accessed 31 Jan 2021

57. Anderson AA. Theory of change tool for strategic planning. The Aspen Institute Roundtable on Community Change. https://www. theoryofchange.org/pdf/tocII_final4.pdf. Published October 2004. Accessed 31 Jan 2021.

58. Allen W. Diagramming a theory of change-Learning for Sustainability. July 2016. https://learningforsustainability.net/post/diagr amming-theory-change/. Accessed 31 Jan 2021.

59. Smith MY, Hammad TA, Metcalf M, et al. Patient engagement at a tipping point - the need for cultural change across patient, sponsor, and regulator stakeholders: insights from the DIA Conference, "Patient Engagement in Benefit Risk Assessment Throughout the Life Cycle of Medical Products." Ther Innov Regul Sci. 2016;50(5):546-53. https://doi.org/10.1177/2168479016662902.

60. Smith SK, Selig W, Harker M, et al. Patient engagement practices in clinical research among patient groups, industry, and academia in the United States: a survey. PLoS ONE. 2015;10(10):e014023210. https://doi.org/10.1371/journal.pone.0140232.

61. Lowe MM, Blaser DA, Cone L, et al. Increasing patient involvement in drug development. Value Health. 2016;19(6):869-78. https://doi.org/10.1016/j.jval.2016.04.009.

62. Janssens R, Huys I, Overbeeke E, et al. Opportunities and challenges for the inclusion of patient preferences in the medical product life cycle: a systematic review. BMC Med Inform Decis Mak. 2019. https://doi.org/10.1186/s12911-019-0875-z.

63. Code of conduct - PARADIGM. 2020. http://imi-paradigm.eu/ petoolbox/code-of-conduct/. Accessed 31 Jan 2021.

64. Patient-Focused Medicines Development (PFMD) Patient Engagement Quality Guidance. https://patientfocusedmedicine.org/peqg/ patient-engagement-quality-guidance.pdf. Published 15 May 2018. Accessed 1 Feb 2021.

65. Englander R, Holmboe E, Batalden P, et al. Coproducing health professions education: a prerequisite to coproducing health care services? Acad Med. 2019;95(7):1006-13. https://doi.org/10. 1097/ACM.0000000000003137.

66. Boudes M, Robinson P, Bertelsen N, et al. What do stakeholders expect from patient engagement: are these expectations being 
met? Health Expect. 2018;21(6):1035-45. https://doi.org/10.1111/ hex.12797.

67. European Medicines Agency (EMA) Regulatory Science to 2025. Strategic reflection. https://www.ema.europa.eu/en/documents/ regulatory-procedural-guideline/ema-regulatory-science-2025strategic-reflection_en.pdf. Accessed 31 Jan 2021.

68. Fujiwara Y. Promoting patient and public involvement in Japan. https://www.pmda.go.jp/files/000235787.pdf. Accessed 31 Jan 2021.

69. ICH harmonised guideline. General considerations for clinical studies E8(R1). Draft version. Currently under public consultation. https://www.ema.europa.eu/en/documents/scientific-guide line/draft-ich-guideline-e8-r1-general-considerations-clinicalstudies-step-2b_en.pdf. Published 8 May 2019. Accessed 31 Jan 2021.

70. Sweeney F. Renovation of ICH guidelines. What is changing and how is EMA contributing? 2020. https://www.ema.europa.eu/en/ documents/presentation/presentation-41-renovation-ich-guide lines-what-changing-how-ema-contributing-f-sweeney_en.pdf. Accessed 31 Jan 2021.

71. Council for International Organizations (CIOMS) Working Group XI: Patient Involvement. https://cioms.ch/working-groups/worki ng-group-xi-patient-involvement/. Accessed 31 Jan 2021.
72. Recommendations on the required capabilities for patient engagement. http://imi-paradigm.eu/petoolbox/pe-capabilities/. Accessed 31 Jan 2021

73. PARADIGM Patient Engagement Toolbox. https://imi-paradigm. eu/petoolbox/. Accessed 31 Jan 2021.

74. Deliverable D6.4. Sustainability of the PARADIGM outputs. PARADIGM WP6 Sustainability Strategy. https://imi-paradigm. eu/wp-content/uploads/2020/09/M30_D6.4-Sustainability-of-thePARADIGM-outputs-1.pdf. Accessed 1 Feb 2021.

75. Patient Engagement Open Forum. https://patientengagementope nforum.org. Accessed 31 Jan 2021.

76. Richards T, Scowcroft H. Patient and public involvement in COVID-19 policy making. BMJ. 2020. https://doi.org/10.1136/ bmj.m2575.

77. EURORDIS Rare Barometer survey "How has COVID-19 impacted people with rare diseases?". https://download2.eurordis. org/rbv/covid19survey/covid_infographics_final.pdf. Published November 2020. Accessed 31 Jan 2021.

78. Pitts PJ, Houÿez F. Patient contribution to the development and safe use of medicines during the Covid-19 pandemic. Ther Innov Regul Sci 2021;55(1):247-249 